\title{
Ultrasonic-assisted Synthesis Of Rod-shaped Hydroxyapatite
}

\author{
Nanoparticles \\ Shi-ting Deng, Hong Yu,Yong-guang $\mathrm{Bi}^{*}$ \\ College of Pharmacy, Guangdong Pharmaceutical University, Guangzhou 510006, \\ Guangdong, China
}

*Corresponding author:biyongguang2002@163.com

Keywords: Ultrasonic-assisted; Hydroxyapatitle; Nanoparticles

Abstract. In this paper, to develop a new method to rapidly synthesize hydroxyapatite nanoparticles as a controlled release carrier, sonochemical method was used to prepare nano-hydroxyapatite (nHAP) without any surfactant and used calcium nitrate tetrahydrate and diammonium hydrogen phosphate as source materials. The composition of obtained hydroxyapatite nanoparticles was determined by X-ray powder diffraction (XRD) and fourier transform infrared spectra (FT-IR). The morphologies and microstructure were observed by transmission electron microscope (TEM). Pure nHAP was successfully synthesized by ultrasonic method without any surfactant. The particles shows not obvious reunion and display rod-shaped structure with length of about 40-120 nm and width of about $30 \mathrm{~nm}$.

\section{Introduction}

Hydroxyapatite (HAP), the mineral composition of human bone tissue, has attracted considerable attention on bone tissue engineering for its biocompatibility and biological activity in recent decades [1, 2]. Additionally, nano-hydroxyapatite (nHAP) have become a research focus in drug carrier systems [3]. Besides, nHAP can improve the permeability of drugs in biological membrane, which is contributed to the percutaneous absorption and thus may improve the bioavailability [4]. However, these good properties would be affected by crystallinity, stoichiometry and specific surface area of nHAP. Therefore, a synthetic route to obtain well-difined nHAP should be taken into consideration.

At present, synthetic routes to obtain nHAP including chemical precipitation method [5], sol-gel method [6], electrochemical deposition method [7] and hydrothermal method [8]. Hydrothermal method may cause extraordinarily time consumption and the waste of reagent. Electrochemical deposition method needs high cost. Therefore, a synthetic routes with advantages oflow cost and efficiency is still need to be investigated. In recent years, ultrasonic method is become a novel technique to obtain nanomaterials [9]. Ultrasonic method can effectively reduce the particle aggregation via cavitation phenomenon that is the formation, growth and severe collapse of cavitation bubble in the liquid medium at the action of ultrasound [10]. In this paper, to develop a new method to rapidly synthesize hydroxyapatite nanoparticles as a controlled release carrier, sonochemical method was used to synthesize nHAP without any surfactant. 


\section{Materials and methods}

Materials. Diammonium hydrogen phosphate, calcium nitrate tetrahydrate and ammonia solution were used to prepare nHAP and obtained from Zhiyuan (Tianjin, China). All aqueous solution were prepared with deionized water and the reagent was without further purification.

The preparation of nHAP. HAP nanoparticles were prepared by ultrasonic systems with ultrasonic horn generates superaudible at a frequency of $25 \mathrm{kHz}$ with the power of 100-950 W (SCIENTZ-IID, Ningbo, China). Firstly, aqueous solutions of diammonium hydrogen phosphate was added into calcium nitrate solution slowly until the $\mathrm{Ca} / \mathrm{P}$ molar ratio reaching about 1.67 . The milky white suspension formed under continuous magnetic stirring and then was treated by ultrasonic irradiation at $280 \mathrm{~W}$ for $30 \mathrm{~min}$. The $\mathrm{pH}$ of reaction mixture was adjusted to 10 with ammonia solution during the whole process. The precipitation reaction was as follows:

$6\left(\mathrm{NH}_{4}\right)_{2} \mathrm{HPO}_{4}+10 \mathrm{Ca}\left(\mathrm{NO}_{3}\right)_{2}+8 \mathrm{NH}_{4} \mathrm{OH}=\mathrm{Ca}_{10}\left(\mathrm{PO}_{4}\right)_{6}(\mathrm{OH})_{2}+20 \mathrm{NH}_{4} \mathrm{NO}_{3}+6 \mathrm{H}_{2} \mathrm{O}$

After finishing the ultrasonic radiation, the reaction mixture was filtered and the residue was washed with purified water and ethanol in sequence. Finally, the residue was dried and subsequently calcined at $700^{\circ} \mathrm{C}$ for 2 hours.

The characterization of nHAP. The composition of nHAP was determined by X-ray powder diffraction (XRD, D8 Advance Diffractometer, Bruker, Germany) with CuK $\alpha_{1}$ radiation. Perkin Elmer Spectrometer 100 device was used to record the fourier transform infrared spectra (FT-IR) from $400-4000 \mathrm{~cm}^{-1}$. The morphologies and microstructure were observed by transmission electron microscope (TEM, FEI Tecnai G2 F20 S-Twin).

\section{Results and discussion}

Figure 1 shows the XRD pattern of obtained product, which shows the major diffraction peak at (200), (111), (002), (102), (210), (211), (300), (202), (301), (310), (222), (213) and (004) plane when compared to the JCPDS data (no. 09-0432). No any impure peaks exists in the above XRD patterns, and diffraction peaks are sharp and clear. It reveals that obtained product is pure nHAP with high intensity.

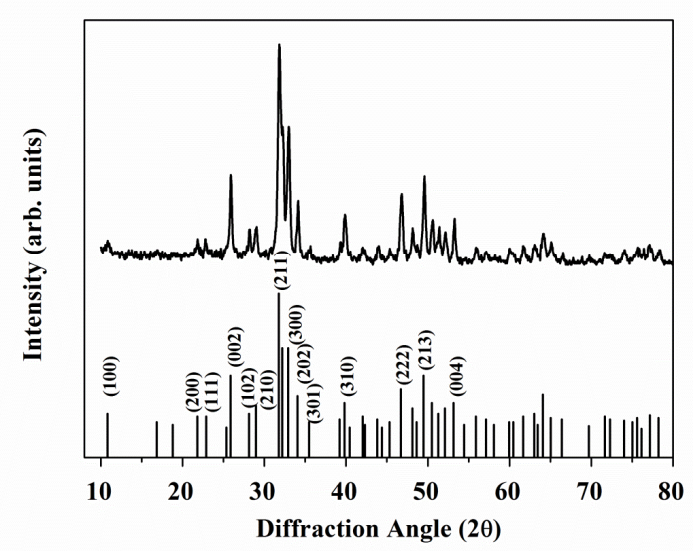

Figure 1. XRD pattern of obtained powder

The FT-IR spectrum of as-synthesized nHAP was shown in Fig. 2. The bands detected at 1091, $1044,964,603,571$ and $473 \mathrm{~cm}^{-1}$ were assigned to vibration peak of $\mathrm{PO}_{4}{ }^{3-}$ group [11]. The hydroxyl stretching vibration of nHAP was located at 3571 and $633 \mathrm{~cm}^{-1}$ [11]. However, weak peaks of carbonate group was also detected at 1455 and $1410 \mathrm{~cm}^{-1}$ [12]. It reveals that obtained nHAP was doped by carbonate ions, due to the carbon dioxide in atmosphere reacted with reaction 
mixture.

The TEM images was shown in Fig. 3. It can be seen from the TEM images, as-synthesised nHAP display a rod-shaped structure with length of about 40-120 nm and width of about $30 \mathrm{~nm}$. Beside, the rod-shaped particles has no obvious reunion. It reveals that the pure nHAP was successfully synthesized by ultrasonic method without any surfactant in a environmental-friendly and effective approach. In addition, the ultrasonic method is less time consumption and reagent waste when compared to the previous reported literatures [13, 14].

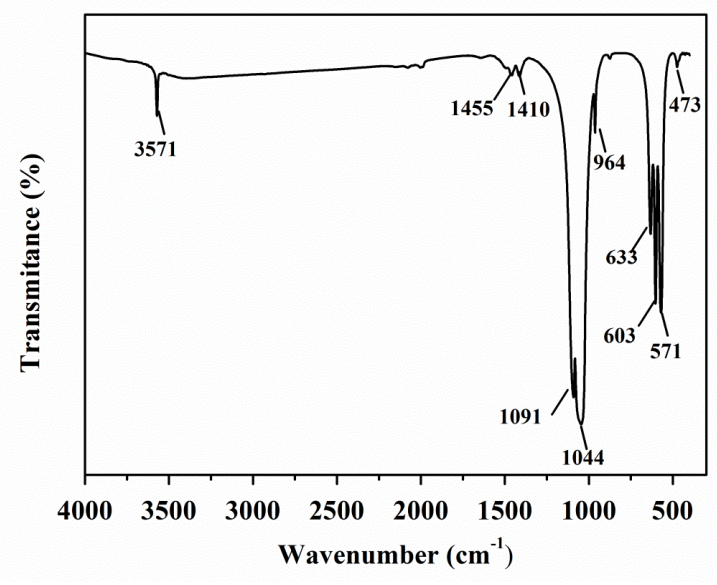

Figure 2. FT-IR spectrum of nHAP

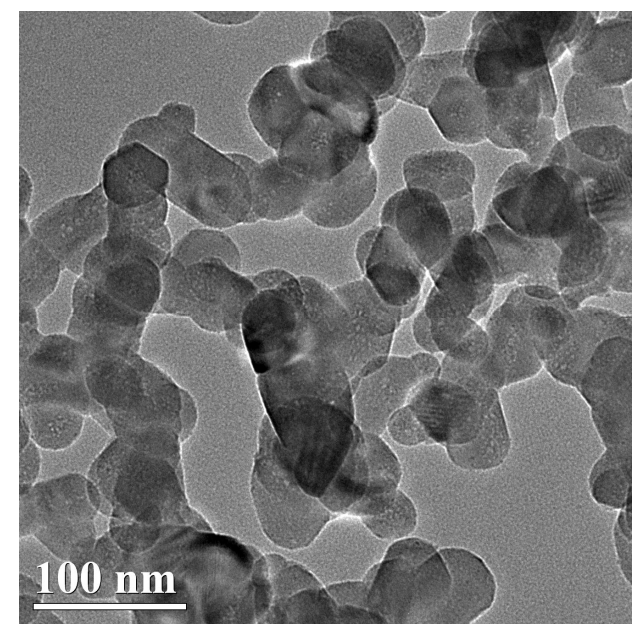

Figure 3. TEM images of nHAP

\section{Conclusions}

The pure nHAP was successfully synthesized by ultrasonic method without any surfactant in a environmental-friendly and effective approach. The rod-shaped hydroxyapatite nanoparticles shows not obvious reunion and the rod-shaped structure with length of about 40-120 nm and width of about $30 \mathrm{~nm}$.

\section{Acknowledgements}

This work was financially supported by Guangdong Provincial Archives Research Project(No. YDK-141-2016). 


\section{References}

[1] S.C. Cox, J.A. Thornby, G.J. Gibbons, M.A. Williams, K.K. Mallick, 3D printing of porous hydroxyapatite scaffolds intended for use in bone tissue engineering applications, Mater Sci Eng C Mater Biol Appl. 47 (2015) 237-247.

[2] C. Sharma, A.K. Dinda, P.D. Potdar, C.-F. Chou, N.C. Mishra, Fabrication and characterization of novel nano-biocomposite scaffold of chitosan-gelatin-alginate-hydroxyapatite for bone tissue engineering, Mater Sci Eng C Mater Biol Appl. 64 (2016) 416-427.

[3] F. Ye, H. Guo, H. Zhang, X. He, Polymeric micelle-templated synthesis of hydroxyapatite hollow nanoparticles for a drug delivery system, Acta Biomater. 6 (2010) 2212-2218.

[4] Y. Wang, X. Wang, K. Wei, N. Zhao, S. Zhang, J. Chen, Fabrication, characterization and long-term in vitro release of hydrophilic drug using PHBV/HA composite microspheres, Mater Lett. 61 (2007) 1071-1076.

[5] I. Mobasherpour, M.S. Heshajin, A. Kazemzadeh, M. Zakeri, Synthesis of nanocrystalline hydroxyapatite by using precipitation method, J Alloys Compd. 430 (2007) 330-333.

[6] O. Kaygili, S.V. Dorozhkin, S. Keser, Synthesis and characterization of Ce-substituted hydroxyapatite by sol-gel method, Mater Sci Eng C Mater Biol Appl. 42 (2014) 78-82.

[7] M.C. Kuo, S.K. Yen, The process of electrochemical deposited hydroxyapatite coatings on biomedical titanium at room temperature, Mater Sci Eng C Mater Biol Appl. 20 (2002) 153-160.

[8] Y. Qi, J. Shen, Q. Jiang, B. Jin, J. Chen, X. Zhang, The morphology control of hydroxyapatite microsphere at high $\mathrm{pH}$ values by hydrothermal method, Adv. Powder Techno. 26 (2015) 1041-1046.

[9] D. Gopi, J. Indira, L. Kavitha, M. Sekar, U.K. Mudali, Synthesis of hydroxyapatite nanoparticles by a novel ultrasonic assisted with mixed hollow sphere template method, Spectrochim Acta A Mol Biomol Spectrosc. 93 (2012) 131-134.

[10] S. Merouani, O. Hamdaoui, Y. Rezgui, M. Guemini, Computational engineering study of hydrogen production via ultrasonic cavitation in water, Int J Hydrogen Energy. 41 (2016) 832-844.

[11] F. Mohandes, M. Salavati-Niasari, Z. Fereshteh, M. Fathi, Novel preparation of hydroxyapatite nanoparticles and nanorods with the aid of complexing agents, Ceram. Int. 40 (2014) 12227-12233.

[12] W.P.S.L. Wijesinghe, M.M.M.G.P.G. Mantilaka, R.M.G. Rajapakse, H.M.T.G.A. Pitawala, T.N. Premachandra, H.M.T.U. Herath, R.P.V.J. Rajapakse, K.G.U. Wijayantha, Urea-assisted synthesis of hydroxyapatite nanorods from naturally occurring impure apatite rocks for biomedical applications, RSC Adv. 7 (2017) 24806-24812.

[13] V. Rodriguez-Lugo, E. Salinas-Rodriguez, R.A. Vazquez, K. Aleman, A.L. Rivera, Hydroxyapatite synthesis from a starfish and [small beta]-tricalcium phosphate using a hydrothermal method, RSC Adv. 7 (2017) 7631-7639.

[14] J. Liu, X. Ye, H. Wang, M. Zhu, B. Wang, H. Yan, The influence of $\mathrm{pH}$ and temperature on the morphology of hydroxyapatite synthesized by hydrothermal method, Ceram. Int. 29 (2003) 629-633. 